\title{
Opportunities and Challenges for Visible Light Communications in $6 \mathrm{G}$
}

\author{
Marcos Katz \\ Centre for Wireless Communications \\ University of Oulu \\ Oulu, Finalnd \\ marcos.katz@oulu.fi
}

\author{
Iqrar Ahmed \\ Centre for Wireless Communications \\ University of Oulu \\ Oulu, Finalnd \\ iqrar.ahmed@oulu.fi
}

\begin{abstract}
This paper discusses the opportunities for visible light communications (VLC) in the future 6G. VLC is a highly attractive optical communication approach for short-range communications, which complements radio. The use of reconfigurable optical-radio networks creates a high performance, highly flexible communication system that can be used in the most stringent environments of $6 \mathrm{G}$. The paper presents and discusses other novel VLC concepts, such as interactive VLC, light-based IoT, living surfaces and optical communications through bio-tissues. These very promising concepts can be used in the identified $6 \mathrm{G}$ verticals, opening new areas of research for $6 \mathrm{G}$.
\end{abstract}

Keywords-optical wireless communications, interactive visible light communications, optical-radio networks, hybrid networks, through-the-tissue optical communications, light-based IoT, living surfaces.

\section{INTRODUCTION}

Currently, at the same time that $5 \mathrm{G}$ is globally introduced, research efforts are increasingly focusing on the definition and further development of its successor, 6G. An international group of experts recently published the first $6 \mathrm{G}$ white paper, a work intended to be updated yearly [1]. At this point, promising concepts and technologies are identified and evaluated. As suggested by the $6 \mathrm{G}$ Flagship Programme [2], the development of $6 \mathrm{G}$ considers not only novel communications technologies, but also highly related areas, such as new materials and components, signal processing, advanced specific applications and others. In principle, 6G integrates short-range, cellular and satellite communications, clearly three different approaches supported by different industries, and defined by different standards. In this paper, the focus is on short-range communications.

Although we are in a very early stage of $6 \mathrm{G}$ development, there are certain general trends expected to have a huge influence on the development of future communications systems. The most notable one is the gradual shift towards higher and higher frequencies. This move is motivated by multiple facts, including the spectral congestion in lower frequency bands (up to $5 \mathrm{GHz}$ ), the need for support of extremely high data rates and others. Fig. 1 summarizes the evolution of spectrum usage for mobile communications, from $1 \mathrm{G}$ to $5 \mathrm{G}$, including some frequency bands likely to be used in $6 \mathrm{G}$. Both, mm-wave as well as $\mathrm{THz}$ bands are currently the focus of intense research by academy and industry communities. The use of these very high frequencies will have a profound impact on system architecture, as at these frequencies the supported range is very small, resulting in cell sizes of reduced dimensions. Components for these radio frequencies need to be carefully designed as their physical size is of the order, or considerably larger than the employed wavelengths. New semiconductors and other materials need to be developed for these operating frequencies. It is expected thus that components for $\mathrm{mm}$-wave and $\mathrm{THz}$ operation will be expensive. In the extreme frequency band, after $\mathrm{THz}$ communications, Fig. 1 also shows the spectrum of light, including infrared as well as visible light. Visible light communications (VLC) have been proposed soon after white light emitting diodes (LEDs) where developed, about two decades ago. Since then, VLC have received extensive attention, mostly by the research community. Today, VLC is a rather mature wireless communications technology, and a highly qualified technology to be used in $5 \mathrm{G}$ and $6 \mathrm{G}$. Optical components have been widely available for decades, and they are typically low-cost. In general terms, propagation in optical wireless communications is quite like that in THz bands, with very short reach and signals being easily blocked by objects.

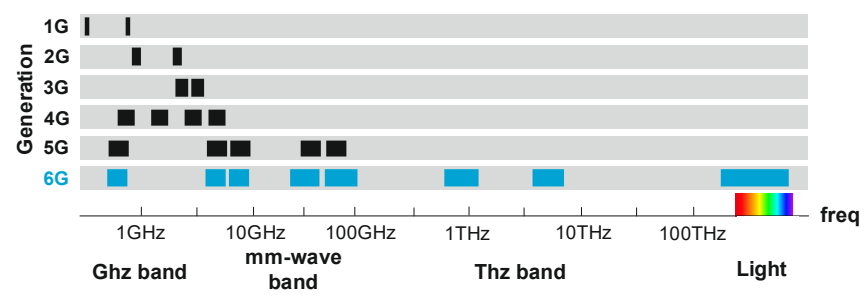

Fig. 1. Spectrum usage throughout past, present and future mobile generations.

The advantages and drawbacks of VLC compared to radio have been discussed widely in the VLC literature. Here we quickly highlight the unique characteristics of VLC, making it a strong technology for $6 \mathrm{G}$ : security, privacy, safety, large amount of unlicensed spectrum, cost and in many cases implementation simplicity. VLC has been proposed and studied widely in the literature, being its main scenario indoors and underwater communications, though vehicular and outdoor scenarios are also quite attractive use cases. In this paper, we position VLC as a key technology for the future $6 \mathrm{G}$ by proposing and number of novel uses and concepts. We include also a literature review considering latest VLC applications.

This paper is organized as follows. Section II presents an overview of advanced VLC usage, beyond conventional indoor applications. Section III introduces a number of novel VLC concepts highly promising for $6 \mathrm{G}$, Section IV discusses briefly the challenges of VLC and Section V concludes the paper.

\section{ADVANCED USES OF VLC}

The development of a new cellular generation has always aimed at creating faster and more reliable solutions, with an 
extended and more sophisticated feature set. Similar expectations are placed on $6 \mathrm{G}$, that it would provide the above-mentioned features along with the massive, ultra-low latency and freedom to define verticals according to the users' requirements. As $5 \mathrm{G}$ will be providing a highly reliable interface interconnecting numerous devices running different applications at very high speed, the quality of service expectations for the upcoming $6 \mathrm{G}$ are quite high. In the near future, the number of interconnected devices is expected to reach billions, resulting in huge amounts of data flowing through the network. In such scenario, $5 \mathrm{G}$ could considerably overcome the challenges of ultra-low latency and high reliability providing the very high bandwidth for communications [3]. As we consider 6G, data speeds requirement will further increase, and the volume of connected devices will be measured by trillions. The extensive features of intelligent networks, capable of handling enormous amount of heterogeneous data in $6 \mathrm{G}$, require ultra-fast communications, and VLC can support $6 \mathrm{G}$ by providing a lightning fast, more secure and safer communication network [4]. VLC has low EMR and radio interference with other devices is not an issue. Even though VLC technology has mostly been developed for indoor scenarios, the technology can be used in a wider range of environments, ranging from underwater to vehicular communications. In $6 \mathrm{G}$, the demand for highly reliable, faster and more secure wireless connectivity calls for novel solutions. One attractive approach is the use of hybrid networks integrating both optical (e.g., VLC) and radio networks. VLC has also been studied for optical backhauls applications as well as for precise local positioning. Many VLC implementations have been demonstrated using off-theshelf equipment, one such application is using commercial cameras to detect numerous users simultaneously [5]. Recently, novel applications of VLC in classrooms for student-teacher communications have been proposed, to enhance the students' learning capabilities and increase student-teacher interaction [6, 20]. Industrial usage of VLC, a promising application area, has not been explored extensively. Manufacturing plants are radio-sensitive environments where VLC can play an important role. A VLC prototype has been tested in [7], the prototype is implemented with a mathematical ARX model to assess the acquired transmission in context to the industrial environment i.e., the environment within the industries is contaminated with fog, smoke, etc. Each of these factors could hamper the transmission capabilities of VLC. Another important VLC application area is healthcare. Today, connected wearables are used for the real-time health monitoring important biomarkers. Since the number of biomarkers has increased, so does the number of devices and required bandwidth. Using $\mathrm{RF}$ could result in electromagnetic interference and could result in loss of important medical information. A novel hybrid VLC and Bluetooth low energy concept has been introduced in [8]. Hybrid networks have been discussed considerable and have proven benefits over single networks. Use of commercial cameras with VLC has also been proposed. While using VLC for body sensors, the location of VLC sensors is critical. A demonstration is presented in [9], where an IR is used as uplink and VLC for downlink. The results show that IR can be used in extreme positions to send data while keeping the overall transmitting power within the safe limit. There is trade-off between transmitting power and data rate. Another novel VLC application is using it for wakeup purposes to start a wireless application on demand [10]. The concept of using solar panel as receiver is quite interesting as it can also serve to harvest energy. The overall energy consumption could then be reduced, and scarce wireless resources can be saved.

The idea of using VLC in aircrafts has been studied where the wireless communication is possible even during take-off and landing. A novel concept of using optical link to establish a wireless connection between two aircrafts has been analysed in [11], where an optical wireless communication (OWC) link has been used as a wireless backhaul to provide an optical link between two aircrafts within a range up to $70 \mathrm{~km}$. A context-aware VLC, proposed in [12], is another novel approach to use resources more efficiently. The time, amount of traffic and location of VLC hotspot can be used to operate a hybrid RF-VLC networks intelligently and efficiently. Gas pipelines are important to watch as there are many factors that need to be monitored continuously, while communication is also required especially between the devices placed in oil wells and those connected at the surface level. A wireless communication solution is presented in [13] using VLC. Optical wireless backhauls have been presented in recent years, these optical backhauls serve as information highway when connecting moving trains. Huge chunks of data can be transmitted using wireless backhauls [14]. Finally, the VLC has also been used to detect the intruders in indoor applications [15].

\section{TOWARDS 6G: NOVEL CONCEPTS AND USES OF VLC}

In this section, we present a number of novel concepts based on the use of VLC technology, considering in particular $6 \mathrm{G}$ and its most stringent requirements.

\section{A. Hybrid Radio-optical Wireless Networks}

Most of the research on VLC is focused to demonstrate the capabilities of optical wireless communications but detached from the fact that radio communication is the dominant way of transferring information wirelessly. The radio community sees VLC as a competing technology, while indeed, optical and radio communications are highly complementary.

The flexibility, scalability and relative simplicity of radio can indeed be combined to the inherent security, safety, privacy of VLC to create a high-performance and robust hybrid communication system. Having wireless devices or nodes equipped with both radio and optical air interfaces allow exploiting the synergy between these systems with no major impact on cost or size. A reconfigurable network selecting dynamically the best operating mode results in a highperformance wireless communication system able to adapt to the radio/optical environment, use case and operating scenario. Such a system is proposed and demonstrated in [1617]. Fig. 2 (left) illustrates the concept, where devices or nodes exploit hybrid radio-optical wireless networks. Different operating modes are dynamically selected by an algorithm according to channel state information (radio/optical), scenario (e.g., local usage policies), user/operator decision, contextual information, etc. Fig. 2 (right) illustrates possible operating modes, according on how optical and radio communications are used in uplink (UL) and downlink (DL). The most basic modes include full optical, full radio and two simple hybrid modes. In diversity mode, both optical and radio 
links are used for UL and/or DL, while the ultra-secure mode multiplexes the signal into radio and optical domains. To be an attractive solution, switching of the modes should be done in a seamless manner, as demonstrated in [17]. Recently, a reconfigurable optical-radio network has been proposed as the key wireless communication network for the hospital of the future [18].

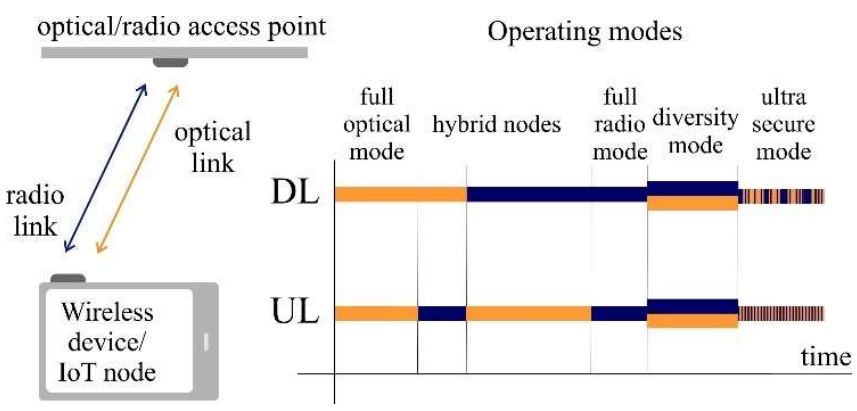

Fig. 2. Hybrid optical-radio networks and their operating modes.

\section{B. Interactive Visible Light Communications}

Key advantages of LEDs are their flexibility and controlability. The speed of how they are LED are controlled defines different application areas, as depicted in Fig. 3. We assume here the use of white RGB LEDs. Slow control speeds, with intensity/color changes in LEDs taking place in a temporal scale from minutes to hours, define smart lighting applicaations, see Fig. 3 (left). The other extreme, Fig. 3 (right), with very high control speeds, defines what we understand by VLC. LEDs are controlled, in this case data modulated, with speeds that can go to hundreds Mbps and beyond. There is however an intermediate operating mode, when LEDs are controlled in a scale of seconds, see Fig. 3 (middle). In such case, we can exploit meaninful visual interaction with users by creating visual signalling aimed at informing, enhancing social interaction, orchestration of situations, guiding and other activities. Light is highly intuitive and conveys directly and quickly information to users. These three operating modes can be combined to create what we defne as interactive visible light communications, iVLC [19].

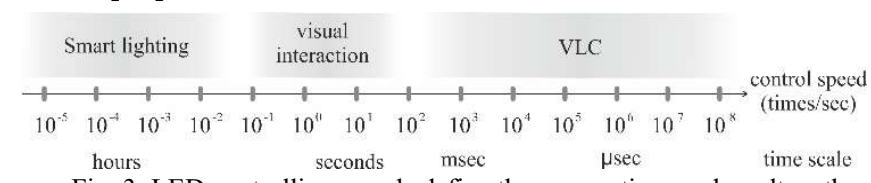

Fig. 3. LED controlling speeds define three operating modes, altogether defining the concept of interactive VLC.

Fig. 4 helps to define the concept of iVLC, showing a generic $N$ x $M$ matrix, in which each element is an RGB LED or group of them. The matrix can be located on the ceiling, wall, floor or in a 3D space, for example. Every element of the matrix can be controlled independently on the following domains, intensity, colour, data as well as space and time. The iVLC concept is a generalization of VLC, providing not only communications capabilities but also lighting as well as visual interaction capabilities, all exploiting the same infrastructure.

There are very many possible applications of iVLC. One of particular interest is its application in the school of the future, where classrooms could be equipped with a light matrix. Smart illumination, student and teacher connectivity as well as an intuitive mean for interact between students and teachers are provided [20]. The latter include visual feedback (at individual, group or classroom level), dynamic work allocation and group work, automatic orchestration of classroom tasks (freezing activities, guiding, warning messages, etc). In addition to educational applications, iVLC can be used at home, office environments, industry, healthcare, and other environments requiring some form of visual interaction. The idea of iVLC also creates the need to investigate and further develop the concept of human-light interaction (HLI), aimed to create interactive effective mechanisms to inform, guide, warn, inspire and motivate people exploiting a light matrix such as shown in Fig. 4.

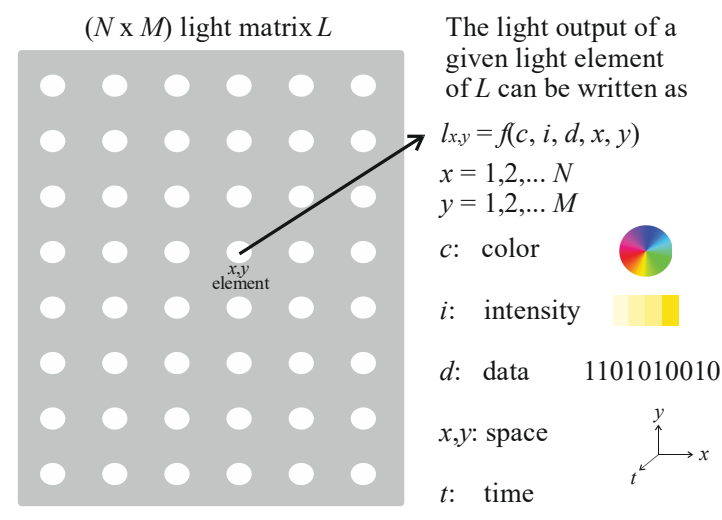

Fig. 4. A light matrix, the principle of iVLC

\section{Light-based Internet of Things}

The concept of Internet of Things (IoT) is slowly but surely connecting the world way beyond people, vehicles and machines. It aims at connecting virtually anything. In the future, trillions of objects could be, among others, identified, controlled and localized remotely. One of the challenges of IoT is that nodes need energy to operate, and while this is not a big issue in many cases (vehicles, home/office appliances, etc.), it is a key restriction in many objects, as internal batteries need to used. The concept of light-based IoT (LIoT) exploits light in multiple ways, namely, to harvest the energy needed to operate, and to transmit and receive wirelessly information [21]. In this way, the node is energy autonomous. This approach is also called Expose and Connect, as nodes are connected to internet whenever they are exposed to light. Even though LIoT can be implemented with discrete/integrated component technologies, this concept becomes highly attractive when implemented with printed electronics (PE). Indeed, PE makes possible to implement the whole system on a substrate like paper or film, with potentially very low cost and negligible environmental impact. Fig. 5 illustrates the concept of LIoT, where a node is connected exploiting light for powering up the system and creating a wireless link. Note that radio can also be used here. Even though PE is not a fully mature technology, key components and technologies needed to implement LIoT are readily available today, such as printed solar cells, printed optical components (photodiodes, LEDs, lenses), printed displays, etc.

The LIoT concept can be extended to consider not only small surfaces but also large areas, such as those of ceilings, floors, walls, furniture and others. This results in the concept of Living Surfaces (LS) [21]. A surface is an opportunity to scavenge energy from the immediate environment and to integrate functionalities on board such as connectivity, signal processing, sensors, displays, actuators and others, all using PE technologies. In case of large surfaces, the harvested energy could be significant, although energy efficiency of 
printed solar cells considerably lags the efficiency of conventional solar cells. LS can have integrated specific functionalities to fulfil certain requirements associated with an application and scenario, or, on the other hand, they could integrate generic functionalities that could be used and reconfigured opportunistically. Functionality diversity or redundancy, e.g., repetition of functions implemented all over a large surface, together with reconfigurable/scalable architectures would make LS a truly powerful and ubiquitous solution in the future. Any surface could become a display, a data processing centre, a sensing area, a wireless connectivity platform, etc. Glimpses of possible applications of LS are shown in a recent $6 \mathrm{G}$ vision video [22].

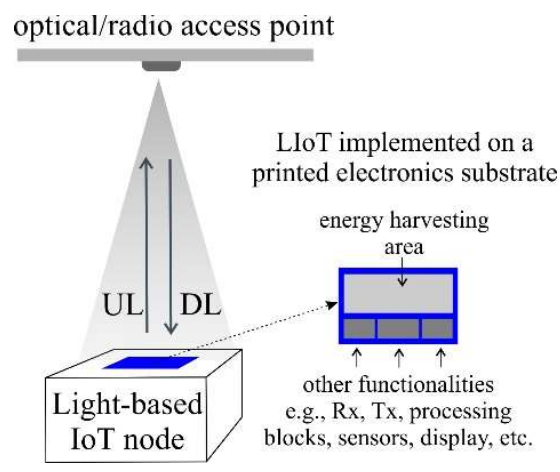

Fig. 5. The concept of light-based IoT (LIoT).

\section{Through The Tissue Optical Communications}

Here we consider a novel application of optical wireless communications for very short ranges, providing a highly secure link for intra-body as well as to/from body communications. Clearly, bio-tissue is the transmission medium, and therefore, near infrared light is used as the optical signal, because such signals propagates the best in the mentioned medium. This application is motivated mostly by security issues. In recent years, research groups have showed that commercial electronics body implants such as pacemakers can be remotely hacked. Today, there exist a great array of implantable devices such as defibrillators medicine dispensers, brain implants, and others. Furthermore, other inbody devices such a sensors and smart pills and ultimately the concept of Internet of Human Body (IoHB) make use of wireless connections. Today, radio and ultrasound techniques are used for the above-mentioned purposes. Radio has in these scenarios issues on security, privacy and safety. Moreover, ultrasound, while safe and secure, is limited by its inherent low data rate support. Fig. 6 illustrates the concept of through the tissue optical communications, where a simplified block diagram of a two-directional optical link is shown.

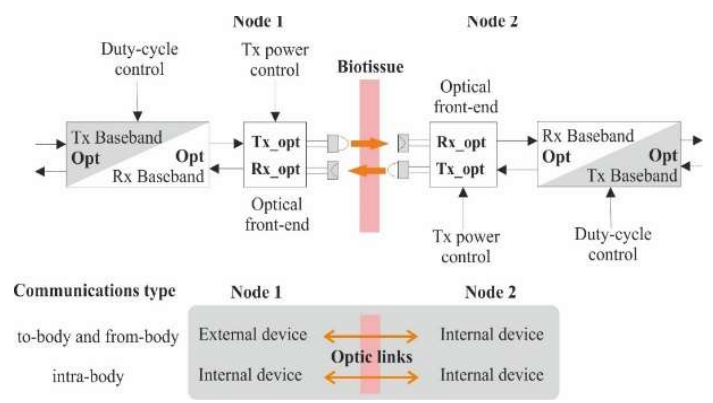

Fig. 6. Principle of through the tissue optical communications.

Through the tissue optical communications is a novel concept and initial experimental results have been encouraging [23]. Preliminary results show that ranges of few centimetres can be achieved, with data rates of several tens of Kbps, using simple and nonoptimized optical links. Advanced modulation schemes, optimized links, MIMO techniques and the used of pulsed communications can be used to increase data throughput and range [24]. Optical phantoms and human bones (e.g., scalp) have been used so far in the experimental setup. Additional measurements in different types of tissues are needed to be able to model the optical channel. This will allow to design optimally the optical link.

\section{VLC AND THE WAY TO 6G}

\section{A. Challenges}

The VLC concept is almost as old as the technology that makes it possible, that is white LEDs. In the twenty or so years of its existency, there have been extensive research as well as experimentals results supporting VLC. However, VLC technology as such has not yet made a breakthrough, and today, its application outside laboratories is quite limited. The key reason for this is not in the technology itself, but it can be atributed to the overwhelming dominance of radio technologies. The optical wireless research community and industry are practically shadowed by the political and economical forces of the well-established century-old radio communities. However, the shift towards $\mathrm{THz}$ bands as well as the incresingly demanding requirements of future communication applications and scenarios in terms of performance, security, privacy and cost/complexity is making VLC an unique candidate for $6 \mathrm{G}$.

\section{B. The Way Forward}

There are currently some efforts to update the already outdated VLC standards (IEEE 802.15.7 from 2011) and to create an extension of IEEE 802.11 to optical communications (IEEE 802.11bb). More than ever, the optical wireless community needs to join forces towards the creation of an attractive concept to be part of $6 \mathrm{G}$. Eventually, short-range communications could be split into $6 \mathrm{G}$-radio and $6 \mathrm{G}$ optical. Novel scenarios and their most stringent requirements will call for nonconventional solutions. For example, underwater and underground (mines) communications. Scenarios where security and privacy are essential, are likely to use VLC solutions. This is the case of hospitals, manufacturing plants, aircrafts, etc. The shift towards higher frequencies has also created global concern on the possible hazards of radio frequency to human in these bands. The introduction of $5 \mathrm{G}$ is already showing, much more intensely than in previous generations, fears and worries by possible users and organizations across the globe. From this perspective, having dual radio-optical communication systems will give users the option of using an RF-free connection. Incorporating such additional optical air interface will certainly give device manufacturers an important competitive edge.

In the last years, research on novel transceiver concepts, new materials and semiconductor structures has made possible the creation of LEDs that can switched at exceptionaly high speeds, and data throughputs on the order of Gbps have been demonstrated at laboratories [25]. This is an important development but not necessarily the only movement needed to make VLC a compelling technology. Further cooperation between the research and industrial community is needed towards the creation of global visions and eventually standards. Moreover, more implementations of 
VLC systems are needed, not necessarily aimed at breaking new speed records but for demostrating systems that work practically well, and that can be implemented with relatively common and unexpensive components. Also, further development and implementation of hybrid radio-optical system will create a fertile ground for the establishment of VLC as one of the key wireless technologies for $6 \mathrm{G}$. In this paper, we have discussed a number of novel concepts and applications for VLC. Note that these can be used in the key verticals identified by the $6 \mathrm{G}$ Flagship program [2], namely automated factories, smart health, automated vehicles as well as personal experiences. The $5 \mathrm{G}$ use scenario triangle highlighting enhanced mobile wideband, massive communications and low latency/high reliability communications will be highly relevant in $6 \mathrm{G}$, as similar goals (though much more stringent) are valid for this future technology. Fig. 7 shows how the VLC concepts discussed in this paper can be used virtually in any scenario of the usage scenario triangle. The use of VLC is not reduced only to niche short-range applications, but it can practically be used the same cases radio is currently used.

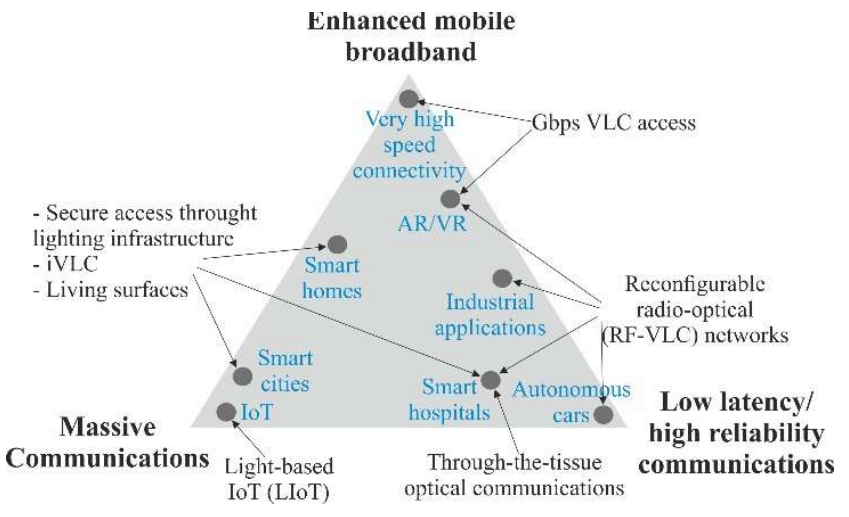

Fig. 7. Application of VLC concepts in the usage scenario triangle.

\section{CONCLUSION}

In this paper, we have discussed the potentials of VLC technology for the upcoming 6G. Unique characteristics of VLC systems were presented. One of the key issues for VLC to become an important player in $6 \mathrm{G}$ is to exploit its synergy with the radio systems to be developed. Several novel VLC concepts and applications with high potential to be used in 6G were also discussed, including reconfigurable opticalradio networks, interactive VLC systems, light-based IoT, living surfaces and optical communications through biotissues. Most of these concepts and applications can be used in the key verticals identified for $6 \mathrm{G}$.

\section{ACKNOWLEDGMENT}

Authors would like to thank the Academy of Finland's support in the project HERONET. Moreover, the support from the 6Genesis Flagship Project is acknowledged.

\section{REFERENCES}

[1] 6G white paper. Available at http://jultika.oulu.fi/Record/isbn978-95262-2354-4

[2] 6G Flagship programme: https://www.oulu.fi/6gflagship/

[3] Selinis, I., Katsaros, K., Allayioti, M., Vahid, S. and Tafazolli, R., 2018. The Race to 5G Era; LTE and Wi-Fi. IEEE Access, 6.

[4] Chowdhury, M.Z., Shahjalal, M., Hasan, M. and Jang, Y.M., 2019. The Role of Optical Wireless Communication Technologies in $5 \mathrm{G} / 6 \mathrm{G}$ and
IoT Solutions: Prospects, Directions, and Challenges. Applied Sciences, 9(20), p.4367.

[5] Hoan, N.C., Hoa, N.V., Luan, V.T. and Jang, Y.M., 2019. Design and Implementation of a Monitoring System using Optical Camera Communication for a Smart Factory. Applied Sciences, 9(23), p.5103.

[6] Apoorv, S., Bhowmick, S.K. and Annadevi, E., 2019, October. Implementation of Li-Fi Technology in Classrooms. In IOP Conference Series: Materials Science and Engineering (Vol. 590, No. 1, p. 012044). IOP Publishing.

[7] Riurean, S., Leba, M., Ionica, A., Stoicuta, O. and Buioca, C., 2019, July. Visible Light Wireless Data Communication in Industrial Environments. In IOP Conference Series: Materials Science and Engineering (Vol. 572, No. 1, p. 012095). IOP Publishing.

[8] Hasan, M., et. al., 2019. Real-Time Healthcare Data Transmission for Remote Patient Monitoring in Patch-Based Hybrid OCC/BLE Networks. Sensors, 19(5), p.1208.

[9] Hoang, T.B., Sahuguede, S. and Julien-Vergonjanne, A., 2019. Optical Wireless Network Design for Off-Body-Sensor Based Monitoring. Wireless Communications and Mobile Computing, 2019.

[10] Carrascal, C., Demirkol, I. and Paradells, J., 2016. On-demand Sensor Node Wake-up Using Solar Panels and Visible Light Communication. Sensors, 16(3), p.418.

[11] Singh, M., 2017. Simulative Analysis of an Inter-aircraft Optical Wireless Communication System Using Amplifier. Journal of Optical Communications, 38(1), pp.1-5.

[12] Du, Z., Wang, C., Sun, Y. and Wu, G., 2018. Context-aware Indoor VLC/RF Heterogeneous Network Selection: Reinforcement Learning with Knowledge Transfer. IEEE Access, 6, pp.33275-33284.

[13] Miramirkhani, F. et al, K., 2018. Visible Light Channel Modeling for Gas Pipelines. IEEE Photonics Journal, 10(2), pp.1-10.

[14] Nakayama, Y., Maruta, K., Tsutsumi, T. and Sezaki, K., 2018. Optically Backhauled Moving Network for Local Trains: Architecture and Scheduling. IEEE Access, 6, pp.31023-31036.

[15] Alsalami, F.M., Ahmad, Z., Zvanovec, S., Haigh, P.A., Haas, O.C. and Rajbhandari, S., 2019. Indoor Intruder Trackin Using Visible Light Communications, Sensors, Volume 19, Issue 20, October, 2019.

[16] Saud M. S. and Katz M., Implementation of a Hybrid Optical-RF Wireless Network with Fast Network Handover, 23th European Wireless Conference; Proceedings of 2017 European Wireless Conference, Dresden, Germany.

[17] Saud A., Hamed I., Kumpuniemi T. and Katz M., Reconfigurable Optical-radio Wireless Networks: Meeting the Most Stringent Requirements of Future Communication Systems, Trans, of Emerging Telecommunications Technologies, Elsevier, pp. 1-15, Jan. 2019.

[18] Ahmed I., Karvonen H., Kumpuniemi T., and Katz., M. Wireless Communications for the Hospital of the Future: Requirements, Challenges and Solutions, International Journal of Wireless Information Networks, Sept. 2019

[19] Katz M., Ahmed I., Laru J. and Häkkinen J. Interactive Visible Light Communications: Supporting Wireless Communications and Smart Visual Interaction with a Common Lighting Infrastructure. South American Colloquium on Visible Light Communications, Nov. 2019.

[20] Laru J., Katz M, Järvelä S and Häkkinen J., Interactive Visible Light Communications: Using Human Light Interaction in Learning Contexts, International Conference on Computer Supported Collaborative Learning, Gothenburg, Sweden, June 7-11, 2015.

[21] Katz M. and O'Brien D., Exploiting Novel Concepts for Visible Light Communications: from Light-based IoT to Living Surfaces, Optik International Journal for Light and Electron Optics 195:163176, DOI: 10.1016/j.ijleo.2019.163176, Aug. 2019

[22] https://www.youtube.com/watch?v=T6ubRoZCeVw\&t=132s

[23] Ahmed I., Bykov A., Popov A., Meglinski I., and Katz M., Optical Wireless Data Transfer Through Biotissues: Practical Evidence and Initial Results, 2019 BodyNets, Florence, Italy, Oct. 2019.

[24] Ahmed I., Bykov A., Popov, A., Meglinski I. and Katz M., Wireless Data Transfer Through Biological Tissues Using Near-infrared Light: Testing Skull and Skin Phantoms. Photonics West 2020, San Francisco, USA, Feb. 1-6. 2020.

[25] Wei Z et al., Gbps Real-Time NRZ-OOK Visible Light Communication System Based on a Packaged Single Layer Quantum Dot Blue MicroLED: First Fabrication and Demonstration, 2019 Asia Communications and Photonics Conference (ACP), 2-5 Nov. 2019. 